\title{
Clonal hematopoiesis in patients with rheumatoid arthritis
}

\author{
Paula Savola ${ }^{1,2}$, Sofie Lundgren ${ }^{1,2}$, Mikko A. I. Keränen ${ }^{1}$, Henrikki Almusa ${ }^{3}$, Pekka Ellonen ${ }^{3}$, Marjatta Leirisalo-Repo ${ }^{4}$, \\ Tiina Kelkka ${ }^{1,2}$ and Satu Mustjoki ${ }^{1,2}$
}

Clonal hematopoiesis $(\mathrm{CH})$ is a phenomenon in which somatic mutations originating from hematopoietic progenitors are detected in peripheral blood cells ${ }^{1,2}$. The prevalence of $\mathrm{CH}$ increases with age, and $\mathrm{CH}$ can be detected in $10-25 \%$ of healthy, elderly individuals ${ }^{1-4}$. Very sensitive methods may allow somatic mutation detection in blood cells in $95 \%$ of $50-60$-year olds ${ }^{5}$.

$\mathrm{CH}$ confers risk for myeloid malignancy and death ${ }^{1,2}$. In addition, recent data has suggested that $\mathrm{CH}$ is also a risk factor for cardiovascular disease ${ }^{1,6,7}$. In mice, Tet2 loss-offunction in myeloid cells alone promoted atherosclerosis and proinflammatory cytokine production ${ }^{6}$. Proinflammatory and dysregulated immune responses play roles in the pathogenesis of multiple diseases. As an example, rheumatoid arthritis (RA) is a chronic autoimmune disease which leads to joint destruction. Despite improved disease outcomes with modern treatments, RA patients have increased risk for death and cardiovascular disease. $\mathrm{CH}$ has not been studied in the context of RA previously, except for one study containing self-reported disease history of arthritis ${ }^{3}$.

Aplastic anemia (AA) and hypoplastic myelodysplastic syndrome (hMDS) are characterized by hypocellular bone marrow and peripheral-blood cytopenias, and are difficult to distinguish ${ }^{8}$. Although $\mathrm{T}$ cells cause hematopoietic stem cell destruction in AA, cytogenetic abnormalities occur in $4-11 \%$, and $\mathrm{CH}$ occurs in up to $50 \%$ of AA cases $^{8}$. AA confers a substantial risk for hematological malignancy ${ }^{8}$.

\footnotetext{
Correspondence: Satu Mustjoki (satu.mustjoki@helsinki.fi)

${ }^{1}$ Hematology Research Unit Helsinki, University of Helsinki and Department of Hematology, Helsinki University Hospital Comprehensive Cancer Center, Helsinki, Finland

${ }^{2}$ Department of Clinical Chemistry and Hematology, University of Helsinki, Helsinki, Finland

Full list of author information is available at the end of the article.
}

In this project, we aimed to characterize $\mathrm{CH}$ in patients with RA. Results were also compared with the data from patients with AA and hMDS, immune-mediated diseases which have established links with $\mathrm{CH}$. In addition, we hypothesized that $\mathrm{CH}$ may modulate chronic inflammation or disease activity in RA. Thus, we compared mutation findings with clinical parameters to investigate associations between $\mathrm{CH}$ and the clinical phenotype in RA.

We collected peripheral blood samples from 59 RA patients who fulfilled the ACR2010 classification criteria for RA and had been monitored in the Helsinki University Hospital rheumatology outpatient clinic after RA diagnosis. Twelve acquired AA and hMDS patients were recruited from the Helsinki University Hospital hematology clinic. We also used samples from two young healthy controls (aged 18-22) as negative controls. All patients gave written informed consent. The ethical board of our institution approved the study and the declaration of Helsinki principles were followed. Due to descriptive nature of our study, no power calculations were performed.

To detect $\mathrm{CH}$, we designed a custom sequencing panel based on Illumina's TruSeq Custom Amplicon technology (Illumina, San Diego, CA, USA). The panel was designed as 250 base-pair amplicons with Illumine Design Studio, and it comprised of 583 amplicons. The panel covered not only genes that are commonly mutated in $\mathrm{CH}$ in healthy individuals but also typically in AA patients. Coding exons of 34 tumor suppressor genes and/or mutational hotspots were sequenced (Supplementary table 1; exact genomic coordinates provided as Supplementary Data). Sequencing was performed with the Illumina HiSeq2500 system with 150 paired-end reads, and the average coverage for each amplicon is shown in the Supplementary figure 1. Peripheral-blood DNA (250 ng) was used for sequencing, 
but for AA/hMDS patients, bone-marrow mononuclear cells (MNC) were used, except for one patient (MDS1, sample type was peripheral blood).

Sequencing data was analyzed by previously described methods ${ }^{9}$. Briefly, sequencing reads were aligned to the Hg19 genome with Bowtie2 and GATK IndelRealigner, but bases with Phred score $<20$ were excluded from further analyses. Variants were required to have sequencing depth $>500$, variant base count $>20$, and comprise over $80 \%$ of all variant bases in the position. Mismapped variants and variants within 5 base-pairs of a 5 base-pair homopolymer were also discarded. Variant with a variant-allele frequency $>35 \%$, population variants with a population frequency of over $1 \%$, and variants that occurred in more than ten individuals were discarded as germline variants. The variants were annotated with the Ensembl Variant Effect Predictor. The Supplementary Material contains more detailed information on variant calling and filtering.

Normal distribution of the data was investigated graphically and with the Shapiro-Wilk test. Statistical tests include two-sided Mann-Whitney test and Fisher's exact test for comparisons between groups. $P$-values $<0.05$ were considered statistically significant. Longitudinal data was analyzed as In-transformed data with a linear mixed model in SPSS using the unstructured covariance type. Sidak correction was used for paired multiple comparisons in the following analyses. Statistical analyses were performed with Graphpad Prism 6 (Graphpad Software, La Jolla, CA, USA) and SPSS Statistics v.23 (IBM, Armonk, NY, USA).

We discovered $\mathrm{CH}$ at $17 \%$ prevalence in $\mathrm{RA}$ and at $33 \%$ in AA/hMDS, which are consistent with previous reports ${ }^{1-4,8}$. The identified mutations fulfilled the criteria for clonal hematopoiesis of indeterminate potential $(\mathrm{CHIP})^{10}$. All synonymous and non-coding mutations were discarded along with mutations with $<2 \%$ variant allele frequency (VAF), because we defined $\mathrm{CH}$ as a process in which mutations provide survival advantage to cells $^{11}$. The $2 \%$ VAF cutoff has also been suggested to define CHIP ${ }^{10}$. We did not require missense mutations to occur in cancer gene databases or have deleterious prediction scores by in silico tools, because these strategies will cause underreporting of novel variants and overreporting of known variants.

In RA patients, DNMT3A mutations were the most common, TET2 mutations ranking second (Figure 1a; Table 1 and Supplementary table 2), consistently with the mutational spectrum found previously in healthy controls $^{1,3,4}$. None of the DNMT3A mutations occurred in the R882 AML/MDS hotspot that has previously been described in $\mathrm{CH}^{1,2,7}$. Frameshift and nonsense mutations comprised 8/12 of all mutations in RA patients (Fig. 1b), and these disruptive mutations had higher VAFs than missense mutations (Fig. 1c; $p=0.0191$ ). This finding suggests that these mutations give survival advantage to the affected clones.

AA/hMDS patients (Supplementary tables 3-4) were characterized by mutations in genes such as LAMB4, PIGA, and STAG2, which differentiates them from RA patients (Fig. 1a) and healthy controls ${ }^{8}$. Consistently with RA patients, most AA patients harbored only one $\mathrm{CH}$ mutation per patient, but one AA patient harbored two $\mathrm{CH}$ mutations (Fig. 1d). Follow-up samples were available from two AA/hMDS patients (AA1 and AA3). One of these patients (AA1) harbored a LAMB4 mutation that occurred in one follow-up sample but disappeared after anti-thymocyte globulin treatment (Supplementary figure 2).

Extremely low VAFs are challenging to detect even with modern sequencing methods. High sequencing coverage is not the only solution to achieve sensitive mutation detection, because sequencing library preparation can induce mutational artifacts, especially $\mathrm{C}>\mathrm{A}$ transversions, to the library DNA ${ }^{12}$. With a VAF cutoff at $2 \%$, half of the singlenucleotide changes were $\mathrm{C}>\mathrm{T}$ mutations (Fig. 1e) in our RA data, supporting the accuracy of mutation calling ${ }^{1-3,11}$.

The prevalence of $\mathrm{CH}$ increased with age in RA patients: the overall prevalence of $\mathrm{CH}$ was $17 \%$ but in $70-79$-year olds it increased up to 25\% (Fig. 1f). However, patients with $\mathrm{CH}$ were not significantly older at sampling (Fig. 1g). Nearly all studied RA patients were treated with antirheumatic drugs before sample collection. The patients' treatment histories included methotrexate in 98\%, hydroxychloroquine in $75 \%$, sulfasalazine in $58 \%$, leflunomide in $10 \%$, and biological drugs in $8.5 \%$ of cases. No differences in the treatment histories were observed between patients with or without $\mathrm{CH}$ (Table 1). Taken together, the signature of $\mathrm{CH}$ in $\mathrm{RA}$ reflects the ageing hematopoietic system with $\mathrm{C}>\mathrm{T}$ transitions and mutations in epigenetic regulators ${ }^{11}$.

As chronic inflammation may cause mutagenesis via DNA damage ${ }^{13}$, we also aimed to explore if $\mathrm{CH}$ is linked with disease severity. In our cohort $(n=59)$ we could not detect any differences in clinical parameters (such as smoking status, serostatus, disease activity at diagnosis or other autoimmune disease) between RA patients with/ without $\mathrm{CH}$ (Supplementary figures 3-4; Supplementary tables 5-6). Similarly, during four years of follow-up, no differences emerged in blood cell indices between RA patients with/without $\mathrm{CH}$ (neutrophil counts and mean corpuscular volume (MCV) are shown in Fig. 1h, i; Supplementary figure 4; Supplementary tables 7-10). This is in line with previous findings in healthy controls, as $\mathrm{CH}$ does not associate with cytopenias in hematologically unselected patients ${ }^{1}$. However, it should be noted that our dataset may lack statistical power to discover subtle changes.

$\mathrm{CH}$ mutations occur mostly in myeloid cells, but mutations in lymphoid cells may also modulate autoimmune responses. We recently discovered $\mathrm{CD} 8+\mathrm{T}$ cells 


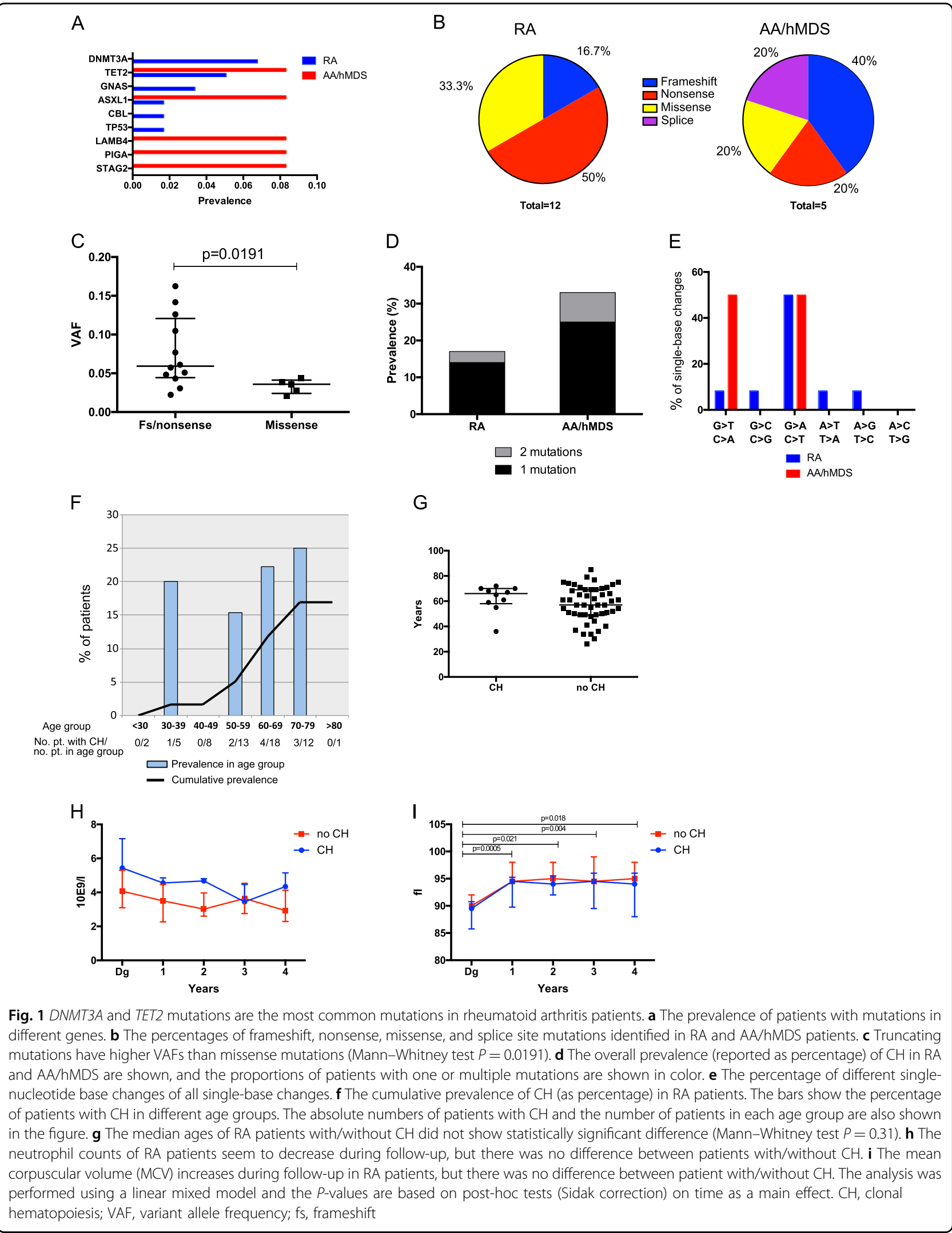




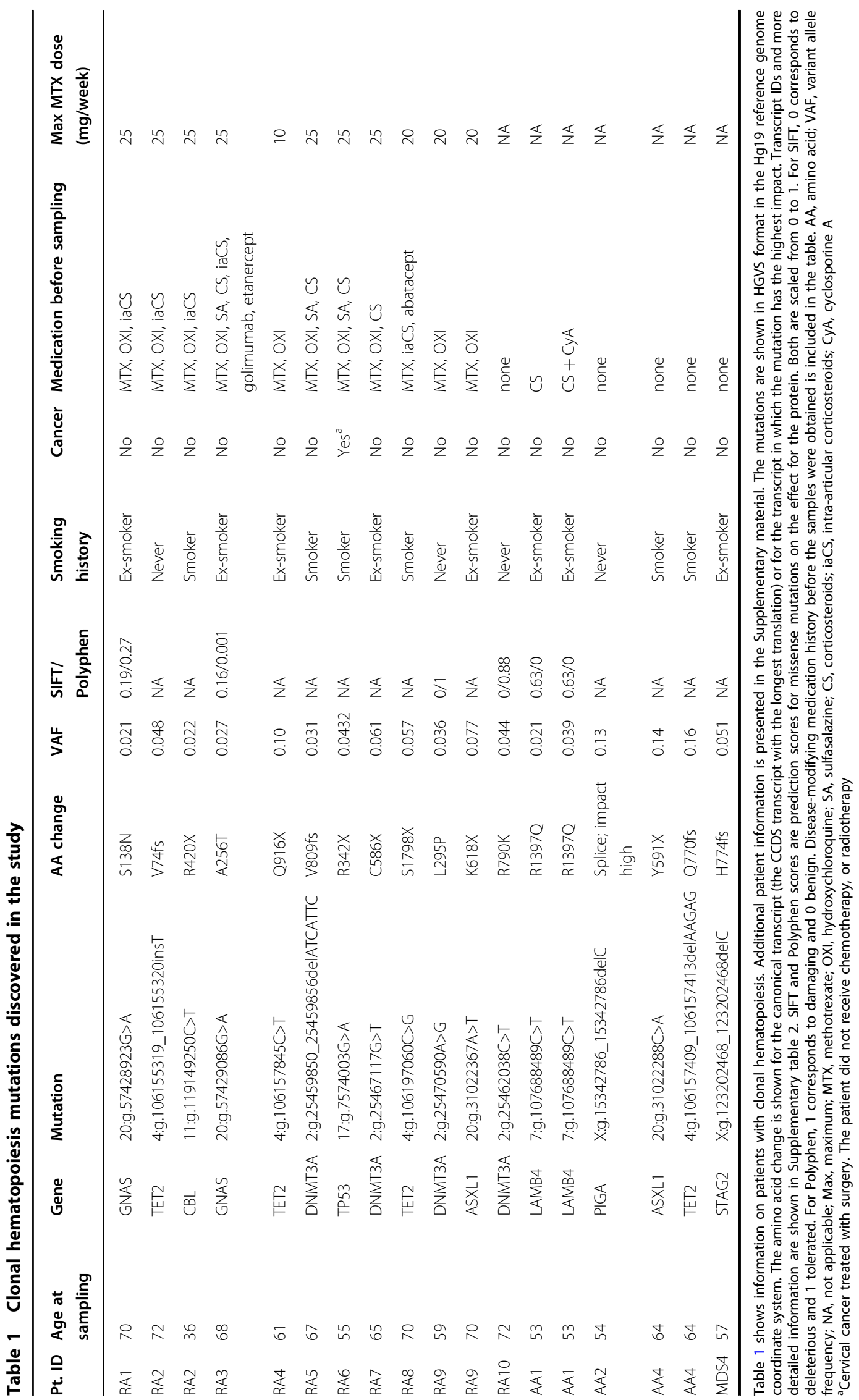


harboring somatic mutations in immune-related genes in RA patients ${ }^{14}$. Similarly, AA- and Felty's syndrome (RA with neutropenia and splenomegaly) patients' CD8+ $\mathrm{T}$ cells harbor somatic mutations ${ }^{9,15}$. Future research is needed to address the "chicken or the egg" dilemma: does autoimmunity increase mutation formation or do mutations promote inflammation and autoimmunity?

Taken together, $\mathrm{CH}$ with a typical mutation profile occurs in RA, but despite of years long systemic inflammation, the rate of $\mathrm{CH}$ is not markedly increased. To our knowledge, this is the first report describing the occurrence of $\mathrm{CH}$ in RA; one previous study included patients with self-reported disease history but did not find a significant association with self-reported arthritis and $\mathrm{CH}^{3}$. However, this data is preliminary, and conclusive results would require analysis of a larger cohort of patients. In addition, although no associations existed between $\mathrm{CH}$ and clinical severity of RA in our data, future studies should assess if a specific mutation or a subset of mutations could impact autoimmunity or treatment responses. This is plausible, as $\mathrm{CH}$ shapes immune responses in other disease contexts: it increases the risk for cardiovascular endpoints ${ }^{1,7}$, and Tet2 deficiency in myeloid cells promotes atherosclerosis and proinflammatory cytokine production in mice 6 . Thus, understanding the functional consequences of all $\mathrm{CH}$-associated mutations, and their roles in various disease conditions, is warranted in the future.

\section{Availability of data and materials}

Due to constraints in the ethical permit, the raw sequencing data of patients is only available from the corresponding author upon reasonable request.

\section{Acknowledgements}

We would like to acknowledge the personnel at Hematology Research Unit Helsinki for their assistance, and the FIMM Technology Center sequencing unit for their assistance on sequencing and data analysis. This work was supported by the European Research Council (M-IMM project), Academy of Finland, Finnish special governmental subsidy for health sciences, research and training, the Sigrid Juselius Foundation, the Instrumentarium Science foundation, the Finnish Cancer Societies, Finnish Cancer Institute, Biomedicum Helsinki foundation, the Finnish medical foundation, Orion research foundation, Juhani Aho foundation, K. Albin Johansson foundation, and Paulo foundation.

\section{Author details \\ ${ }^{1}$ Hematology Research Unit Helsinki, University of Helsinki and Department of Hematology, Helsinki University Hospital Comprehensive Cancer Center, Helsinki, Finland. ${ }^{2}$ Department of Clinical Chemistry and Hematology, University of Helsinki, Helsinki, Finland. ${ }^{3}$ Institute for Molecular Medicine Finland (FIMM), HILIFE; University of Helsinki, Helsinki, Finland. ${ }^{4}$ Rheumatology, University of Helsinki and Helsinki University Hospital, Helsinki, Finland}

\section{Authors contributions}

P.S., S.L., M.A.I.K., P.E., T.K. and S.M. designed the study and experiments. P.S., M.A.I.K. and M.L.-R. recruited the patients. P.S., T.K., S.L. and M.A.I.K. collected the samples. P.S., S.L., P.E. and H.A. analyzed the data. P.S. and S.M. wrote the manuscript. All authors contributed to writing and approved the manuscript.

\section{Author details}

${ }^{5}$ Hematology Research Unit Helsinki, University of Helsinki and Department of Hematology, Helsinki University Hospital Comprehensive Cancer Center, Helsinki, Finland. ${ }^{6}$ Department of Clinical Chemistry and Hematology, University of Helsinki, Helsinki, Finland. ${ }^{7}$ Institute for Molecular Medicine Finland (FIMM), HILIFE; University of Helsinki, Helsinki, Finland. ${ }^{8}$ Rheumatology, University of Helsinki and Helsinki University Hospital, Helsinki, Finland

\section{Conflict of interest}

S.M. has received honoraria and research funding from Novartis, Pfizer, and Bristol-Myers Squibb (not related to this study). The remaining authors declare no competing interests.

\section{Publisher's note}

Springer Nature remains neutral with regard to jurisdictional claims in published maps and institutional affiliations.

Supplementary Information accompanies this paper at (https://doi.org/ 10.1038/s41408-018-0107-2)

Received: 31 May 2018 Revised: 8 June 2018 Accepted: 15 June 2018 Published online: 26 July 2018

\section{References}

1. Jaiswal, S. et al. Age-related clonal hematopoiesis associated with adverse outcomes. N. Engl. J. Med. 371, 2488-2498 (2014).

2. Genovese, G. et al. Clonal hematopoiesis and blood-cancer risk inferred from blood DNA sequence. N. Engl. J. Med. 371, 2477-2487 (2014).

3. Buscarlet, M. et al. DNMT3A and TET2 dominate clonal hematopoiesis and demonstrate benign phenotypes and different genetic predispositions. Blood 130, 753-762 (2017).

4. Acuna-Hidalgo, R. et al. Ultra-sensitive sequencing identifies high prevalence of clonal hematopoiesis-associated mutations throughout adult life. Am. J. Hum. Genet. 101, 50-64 (2017).

5. Young, A. L., Challen, G. A., Birmann, B. M., \& Druley, T. E. et al. Clonal haematopoiesis harbouring AML-associated mutations is ubiquitous in healthy adults. Nat. Commun. 7, 12484 (2016).

6. Fuster, J. J. et al. Clonal hematopoiesis associated with Tet2 deficiency accelerates atherosclerosis development in mice. Science. 355, 842-847 (2017).

7. Jaiswal, S. et al. Clonal hematopoiesis and risk of atherosclerotic cardiovascular disease. N. Engl. J. Med. 377, 111-121 (2017).

8. Ogawa, S. CME article clonal hematopoiesis in acquired aplastic anemia. Blood 128, 337-348 (2016).

9. Savola, P. et al. Somatic STAT3 mutations in Felty syndrome: an implication for a common pathogenesis with large granular lymphocyte leukemia. Haematologica 103, 304-312 (2018).

10. Steensma, D. P. et al. Clonal hematopoiesis of indeterminate potential and its distinction from myelodysplastic syndromes. Blood 126, 9-16 (2015).

11. Bowman, R. L., Busque, L. \& Levine, R. L. Clonal hematopoiesis and evolution to hematopoietic malignancies. Cell Stem Cell 22, 157-170 (2018).

12. Costello, M. et al. Discovery and characterization of artifactual mutations in deep coverage targeted capture sequencing data due to oxidative DNA damage during sample preparation. Nucleic Acids Res. 41, e67 (2013).

13. Nakad, R. \& Schumacher, B. DNA damage response and immune defense: links and mechanisms. Front. Genet. 7, 147 (2016).

14. Savola, P. et al. Somatic mutations in clonally expanded cytotoxic T lymphocytes in patients with newly diagnosed rheumatoid arthritis. Nat. Commun. 8, 15869 (2017).

15. Jerez, A. et al. STAT3-mutations indicate the presence of subclinical T cell clones in a subset of aplastic anemia and myelodysplastic syndrome patients. Blood 122, 2453-2459 (2013). 